\title{
Tuning and Sensitivity Improvement of Bi-metallic Structure-based Surface Plasmon Resonance Biosensor with 2-D $\xi$-Tin Selenide nanosheets
}

Natarajan Sathya

Engineering Department, Scientific Society Group, Tamilnadu

Bhishma Karki

Tribhuvan University - Tri-Chandra Multiple Campus

Kantilal Pitambar Rane

KCE COEM Jalgaon

Ankit Jha

DIT University

Amrindra Pal ( $\nabla$ amrindra.ieee@gmail.com )

DIT University Dehradun https://orcid.org/0000-0001-7806-2453

\section{Research Article}

Keywords:

Posted Date: July 15th, 2021

DOl: https://doi.org/10.21203/rs.3.rs-620951/v1

License: (9) This work is licensed under a Creative Commons Attribution 4.0 International License. Read Full License 


\section{Abstract}

This manuscript aims to visualize the effect of tin selenide ( $\mathrm{SnSe}$ ) on the sensing application of the SPR biosensors. Tin selenide is the 2-Dimensional material transition metal dichalcogenide family element. By utilizing the extraordinary properties of the SnSe, high sensitivity and high performance of the biosensor is analyzed by utilizing the extraordinary properties of the SnSe. A unique two-dimensional substantial heterostructure, $\epsilon$-tin selenide ( $\mathrm{SnSe}$ ) /graphene layer has been deposited over the metal surface to improve the sensitivity; moreover, the sensitivity is limited to a certain extent. The $\epsilon-\mathrm{SnSe}$ nanosheet is placed in between two layers of gold ( $\mathrm{Au}$ ) in the Kretschmann arrangement. The proposed configuration has a maximum sensitivity of $214^{\circ} / \mathrm{RIU}$, which is $93.81 \%$ higher than the conventional sensor. The performance parameters like FWHM, detection accuracy and quality factor have been analysed. The $\epsilon-\mathrm{SnSe}$ material is an air-stable 2-D nanosheet and has application in chemical, medical, and biological sensors.

\section{Introduction}

In the last few decades, the tremendous development of Surface Plasmon Resonance (SPR) technology has been reported for biomedical applications. Detecting cancer cells, DNA Hybridization, antibody characterization, protein conformation, and the recent detection of the CORONA virus has proved that the technique is more beneficial for real-time applications. In the biosensing field, to detect, analyze and characterize the biomolecule, chemical, environment, and food [1]-[5], these biosensors are highly sensitive and facilitate real-time analysis of the contaminated analyte [6]-[8]. The SPR biosensor works on the phenomenon of the shift of resonance dip. As the refractive index of the sensing layer varies [9], [10], a sharp dip in the resonance curve is obtained due to the adsorption of the incident light in the sensing medium. The Kretschmann configuration is the most acceptable configuration for designing the biosensor [11], [12]. This configuration has a and has a thin layer of metal. The layer of metal is used to absorb the biomolecules or chemical molecules. The metal has poor adsorbability, so few layers of 2dimensional material is associated with enhancing the biosensors' performance [13]-[17]. When an incident light beam propagates through two media interfaces with the different refractive indexes at a particular angle and suitable dielectric constants (typically a metal with high concentration of free electrons and a dielectric medium), the Surface Plasmon gets excited [18].

Single-layer SnSe also possesses fantastic electrical and optical properties and generates high interest as a $2 \mathrm{D}$ material beyond the predecessor members, which displays the same structure as

graphene and phosphorene [19]-[21]. As a classical p-type IV-VI semiconductor, SnSe has a narrow gap [22]. That SnSe has a layered crystal structure similar to those of other IV-VII binary semiconductors, such as $\mathrm{SnSe}, \mathrm{GeS}, \mathrm{GeSe}$, etc. In experiments, the assumption that unilaminar crystal SnSe would display different thermoelectric properties on different axes was authenticated by Zhao et al. [16], who manufactured hole-doped single-crystal SnSe [18]. A lot of SnSe allotropes have been investigated by

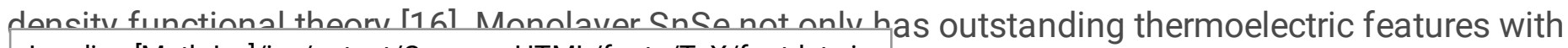
Loading [MathJax]/jax/output/CommonHTML/fonts/TeX/fontdata.js 
semiconductor properties, but is also an eminent optoelectronic material, but there are few systematic studies on the optic and electric characteristics of SnSe allotropes. A metal like Gold (Au) is not susceptible to oxidation and does not react with most chemicals; and hence it is often used as the metal film in sensors. At the metal-dielectric interface, the intensity of the incident light beam reaches a maximum and decays exponentially into both the medium [23]. Since the metal and wave interaction is lossy, the EM Field of a Surface Plasmon is concentrated in a vast majority in the dielectric medium close to the metal surface. The SPR phenomenon results in a graded reduction in the intensity of the reflected light. Surface Plasmon Resonance (SPR) sensors have generated considerable interest since they allow real-time detection of biomolecular interactions, rapid, level free, and valuable diagnostic tool for diseases having virus size of the order of nanometer or less [24].

In particular, graphene has attractive properties such as tunable electrical \& optical characteristics [25], ring-type carbon structure \& high surface-to-volume ratio. Thus the adsorbates can easily interact with this structure, increasing the adsorption suitable for biosensors [26], [27].

Enhancing the sensor's sensitivity is an emerging issue and is being studied at different places; different methods and strategies have been proposed. Conventionally Gold ( $\mathrm{Au}$ ) or Silver (Ag) having high free electrons closed to the boundary was chosen, and superimposed over it a dielectric medium of finite thickness helped in generating charge density oscillation at the metal-dielectric interface when monochromatic light was incident over it [28]. SPR technique help in estimating the dimension of DNA [29], RNA or Blood cells which cannot be measured directly due to its sizes less than nanometer; therefore, the indirect measurement technique, which was evolved in 1968 by Kretschmann, opened a new avenue where the refractive index variation interacting with incident light photon and the reflected ray thus quantify the size or the nature of the virus under investigation. The binding of the legend differs; therefore, SPR has successfully detected the diverse effect and resulting refractive index change, for example protein- protein and DNA-DNA etc. [30], [31]. SnSe can enhance the sensitivity of the biochemical sensors. It is an indirect bandgap 2-D semiconductor material, stable in the air.

The manuscript is sectioned as follows, Sect. 2 proposed theoretical model and design consideration for the designed biosensor. Section 3 consists of the results and discussion, and finally, Sect. 4 concludes the proposed work.

\section{Theoretical Model And Design Consideration}

We consider a modified biosensor configuration, as shown in Fig. 1. Each layer of the materials is assumed to be stacked along the z-axis. BK7 is chosen as a coupling prism in our structure, which is covered with one layer of gold-1, $\epsilon-\mathrm{SnSe}$ layer is close to gold-1 layer, and the other side of $\epsilon-\mathrm{SnSe}$ layer also connects with another gold-2 layer. Graphene layer is used as a biomolecular recognition element, which is close to the gold-2 layer. Besides, the thickness of the two layers of gold is not the same. We set the thickness of two layers of gold as $W_{1}=40 \mathrm{~nm}$ and $W_{3}=20 \mathrm{~nm}$, respectively. The

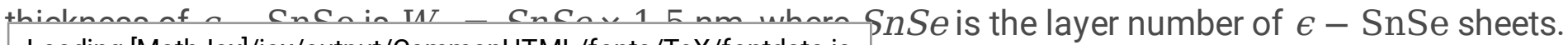


Graphene is connected with the sensing layer for increasing the ability of macromolecular adsorption of the biosensor, and the thickness of graphene is $W_{2}=G \times 0.34 \mathrm{~nm}$, where $G$ is the layer number of graphene sheets. A TM-polarized light is assumed to be incident from one side of the prism and receive reflected light from the other side of the prism. The received data can be used to calculate the reflectivity and sensitivity of the structure.

The refraction index $\left(n_{\text {Prism }}\right)$ of BK7 layer is expressed as [32]

$$
n_{\text {Prism }}=\left(\frac{1.03961212 \lambda^{2}}{\lambda^{2}-0.00600069867}+\frac{0.231792344 \lambda^{2}}{\lambda^{2}-0.0200179144}+\frac{1.0104694 \lambda^{2}}{\lambda^{2}-103.560653}+1\right)^{\frac{1}{2}}
$$

1

where $\lambda$ is the wavelength of the optical signal.

The RI of the analyte/metal can be computed using the Drude model [33] by the formula:

$n_{1,3}(\lambda)=\sqrt{1-\frac{\lambda^{2} \lambda_{c}}{\lambda_{p}^{2}\left(\lambda_{c}-i \lambda\right)}}$, where $\lambda_{p}$ and $\lambda_{c}$ are the wavelengths of silver for plasma and collision and are taken as $1.6826 * 10^{-7} \mathrm{~m}$ and8.9342 $* 10^{-5} \mathrm{~m}$, respectively [34].

At $\lambda=633 \mathrm{~nm}$ the refractive index of $\epsilon-\mathrm{SnSe}$ is considered as $4.4+3.53 \mathrm{i}$ [23] in the visible region. In the proposed multi-layered structure, reflectivity is calculated by formula as follows:

$$
r_{p m_{1} d_{1} m_{2} d_{2}}=\frac{r_{p m_{1}}+r_{m_{1} d_{1} m_{2} d_{2}} e^{2 i K m_{1} x d m_{1}}}{1+r_{p m_{1}} r_{m_{1} d_{1} m_{2} d_{2}} e^{2 i K m_{1} x d m_{1}}}
$$

2

where

$$
K_{i X}=\sqrt{\left(\frac{2 \Pi}{\lambda}\right)^{2} \epsilon_{i}-K_{Z}^{2}} i=\text { prism }_{\text {metal }}, \text { dielectric }_{1}, \text { metal }_{2}, \text { dielectric }_{2}
$$

3

$$
r_{\text {prismmetal }}=\frac{\left(\epsilon_{\text {metal }} K_{\text {prismx }}-\epsilon_{\text {prism }} K_{\text {metalx }}\right)}{\left(\epsilon_{\text {metal }} K_{\text {prismx }}+\epsilon_{\text {prism }} K_{\text {metalx }}\right)}
$$




$$
r_{\text {metaldielectric }}=\frac{\left(\epsilon_{\text {dielectric }} K_{\text {metalx }}-\epsilon_{\text {metal }} K_{\text {dielectricx }}\right)}{\left(\epsilon_{\text {dielectric }} K_{\text {metalx }}+\epsilon_{\text {metal }} K_{\text {dielectricx }}\right)}
$$

The condition for resonance is fulfilled using the Eqs. 2 and 3. The equation computes the sharp downfall in the resonance curve:

$$
K_{z}=K_{s p}=\frac{2 \pi}{\lambda} n_{0} \operatorname{Sin} \theta_{S P R}=\operatorname{real}\left(\frac{2 \pi}{\lambda} \sqrt{\frac{\epsilon_{m} \epsilon_{s}}{\epsilon_{m}+\epsilon_{s}}}\right)
$$

\section{4}

where $n_{0}$ is the RI of the sensing layer, $\epsilon_{s}$ and $\epsilon_{m}$ is the dielectric constant for the sensing layer and analyte/Metal, $\theta_{S P R}$ is resonance angle and $K_{S p}$ is propagation constant. The RI of the graphene film is given as $n_{5}=3+i \frac{C}{3} \lambda$, where $C=5.44 \mu m^{-1}$ [20]. The RI of sensing film is taken as $n_{s}=1.373$. The sensitivity and detection accuracy are the performance parameters of the biosensor, and the same is computed for the proposed biosensor. The sensitivity of the biosensor is represented as $S=\delta \theta_{S P R} / \delta n$, where $\delta n$ is the change in the RI. The detection accuracy (DA) of the biosensor can be expressed as $D A=1 / F W H M$, where FWHM is the width of the spectra of the SPR curve at that point, the reflectivity is $50 \%$ of the maximum value. The high value of the sensitivity and DA is desirable [35]. The quality factor can be expressed as $Q=S . D A=S / F W H M$, directly depends on the FWHM and sensitivity. Table 1 shows the optimized parameters selected for the theoretically examined biosensor.

Table 1

\begin{tabular}{|c|c|c|c|c|}
\hline \multirow[t]{2}{*}{ Film of the materials } & \multirow[t]{2}{*}{ Used material } & \multicolumn{2}{|c|}{$\mathrm{RI}$ of the material at $633 \mathrm{~nm}$} & \multirow[t]{2}{*}{ Thickness (nm) } \\
\hline & & Real part (n) & Imaginary part (k) & \\
\hline Layer I & Prism BK7 & 1.5151 & - & - \\
\hline Layer II & Au (Metal) & 0.18377 & $3.4313 i$ & $w_{1}=40$ \\
\hline Layer III & $\epsilon-$ Tin Selenide & 4.4 & $3.53 \mathrm{i}$ & $w_{2}=M^{*} 1.5$ \\
\hline Layer IV & Au (Metal) & 0.18377 & 3.4313 & $w_{3}=20$ \\
\hline Layer V & Graphene & 2.4105 & - & $w_{4}=0.34 * N$ \\
\hline
\end{tabular}

Optimized parameters for the proposed biosensor

\section{Results And Discussions}

Emphasis has been made to improve the sensitivity and performance of biosensor low refractive index Loading [MathJax]/jax/output/CommonHTML/fonts/TeX/fontdata.js 
refractive index, it provides the sharp dip in the resonance curve compared to the low refractive indices' prism. Low refractive prism provides better values of the FWHM, angle of resonance, the shift in the resonance curve, and sensitivity than the high refractive index's prism. To get the high sensitivity and FWHM, the flat end of the prism is polished with a 40nm thick gold layer. On the top of the Au layer, few layers of the SnSe are deposited to examine the structure's sensitivity. Another Au layer of $20 \mathrm{~nm}$ is deposited over the SnSe layer to enhance the sensor's sensitivity. A single metal layer cannot increase sensitivity too much, so using bimetallic layer is used. In the end, the graphene layer is spread over the gold-1 layer.

To extend the discussion, a single layer of the graphene is present, and the SnSe layer is missing in the structure, i.e., $\mathrm{SnSe}=0, G=1$. Now the impact of the graphene is analyzed, and it is found that offset resonance dip and sensitivity both are improvised and values of $\delta \theta_{S P R}=0.99^{\circ}$ and $198^{\circ} / \mathrm{RIU}$ are claimed respectively (shown in Fig. (c)). The enhanced sensitivity and the increase in resonance curve offset dip in this structure are attributed to the higher adsorption rates due to the large surface area and rich $\pi$ conjugation structure offered by graphene, making it the appropriate choice for dielectric top layer SPR sensing applications [25], [36].

Another scenario was evaluated for the impact by introducing a single layer of SnSe and a monolayer of graphene in the biosensor ( $\mathrm{SnSe}=1, G=1$ ). This results in significant shifts and a dip in the resonance curve. The resonance angle was obtained as $\delta \theta_{S P R}=1.02^{\circ}$, whereas the sensitivity was $214^{\circ} /$ RIU as depicted in Fig. 2 (d). This comparative study reveals that the resonance angle offset increases with the addition of a single layer of graphene, a single layer of SnSe, compared to the conventional sensor. The best results were obtained by adding a single layer of graphene $\&$ a single layer of SnSe. Table 2 consists of performance parameters that are directly obtained from the theoretical study of the proposed work. Based on our knowledge, few parameters are better reported than the previously existing literature.

Table 2

Final values of the different parameters for the sensor

\begin{tabular}{|c|c|c|c|c|c|c|c|}
\hline Structures (Prism BK7) & $\begin{array}{l}\text { Resonance } \\
\text { Angle }\left(\theta_{\mathrm{SPR}}\right)\end{array}$ & $\delta \theta$ & FWHM & Sensitivity & $\begin{array}{l}\text { Quality } \\
\text { Factor }\end{array}$ & DA & $\mathbf{R}_{\min }$ \\
\hline 1. Bimetallic layer (Au) & 76.86 & 0.97 & 4.434 & 194 & 46.01 & 0.23 & 0.22 \\
\hline $\begin{array}{l}\text { 2. Bimetallic layer }+ \\
\epsilon-\mathrm{SnSe}\end{array}$ & 77.42 & 0.98 & 7.039 & 196 & 30.12 & 0.18 & 030 \\
\hline $\begin{array}{l}\text { 3. Bimetallic layer }+ \\
\text { Graphene }\end{array}$ & 77.44 & 0.99 & 5.41 & 206 & 38.08 & 0.14 & 0.43 \\
\hline $\begin{array}{l}\text { 4. Bimetallic layer }+ \\
\text { Graphene }+\epsilon-\text { SnSe }\end{array}$ & 78.02 & 1.02 & 7.947 & 214 & 26.93 & 0.13 & 0.49 \\
\hline
\end{tabular}

By comparing the relevant data, we can find that the resonance angle offset of the biosensor in our the addition of graphene and the $\epsilon-\mathrm{SnSe}$ 
layer. The traditional SPR structure contains only a prism and gold layer. Hence, we can conclude that the sensitivity of biosensors in our structure has significantly improved compared with the traditional design by adding graphene and $\epsilon-$ SnSe layer.

From the analyses made above, it is clear that the refractive index of the sensing layer can control the sensitivity of the biosensor. To better understand the enhancement of the sensitivity of the biosensor by graphene and $\epsilon-$ SnSe layers, we also plot the dependence of the sensitivity of the biosensor on the refractive index of the sensing layer, as shown in Fig. 3. Here we select the sensing layer refractive index range from 1.368 to 1.373 . It can be seen that as the refractive index of the sensing layer increases from 1.368 to 1.373 , the sensitivity increases significantly.

Table 3

Depicts the analytical values at which the quality factor obtained is maximum

\begin{tabular}{|llllll|}
\hline LAYERS & FWHM & $\begin{array}{l}\text { DETECTION } \\
\text { ACCURACY }\end{array}$ & SENSITIVITYY & $\begin{array}{l}\text { QUALITY } \\
\text { FACTOR }\end{array}$ & $\begin{array}{l}\text { MINIMUM } \\
\text { REFLECTANCE }\end{array}$ \\
\hline $\begin{array}{l}\mathrm{SnSe}=1 \mathrm{G} \\
=1\end{array}$ & 7.947 & 0.1258 & 214 & 26.9 & 0.487 \\
$\begin{array}{l}\mathrm{SnSe}=2 \mathrm{G} \\
=1\end{array}$ & 9.774 & 0.1023 & 196 & 20.1 & 0.6077 \\
$\begin{array}{l}\mathrm{SnSe}=1 \mathrm{G} \\
=2\end{array}$ & 8.798 & 0.1137 & 212 & 24.1 & 0.5376 \\
\hline
\end{tabular}

Table 3 comprises the performances of the biosensor for the maximum change in the $\mathrm{Rl}$ is 0.005 , the thickness of the Au layers is chosen $40 \mathrm{~nm}$ and $20 \mathrm{~nm}$, the thickness of the $\epsilon-\mathrm{SnSe}$ is $1.5 \mathrm{~nm}$, and thickness of the graphene 0.34 . Table 4 reports the comparative analysis of the earlier published work. Table 4 reflects that all the referenced work is published, and it can consider that all are standard. The proposed work here shows the best performance parameters as well as high sensitivity.

Table 4

Comparative analysis with the earlier reported work

\begin{tabular}{|llll|}
\hline Layers & Wavelength $\lambda$ & $\begin{array}{l}\text { Sensitivity } \\
\text { (deg/RIU) }\end{array}$ & Reference \\
\hline $\begin{array}{l}\text { Chromium, Gold, SnSe nanosheet, sensing } \\
\text { layer }\end{array}$ & $633 \mathrm{~nm}$ & 160 & [37] \\
\hline Silver, SnSe, sensing layer & $633 \mathrm{~nm}$ & 154 & {$[23]$} \\
\hline Gold, SnSe, Gold, graphene, sensing layer & $633 \mathrm{~nm}$ & 214 & $\begin{array}{l}\text { Proposed } \\
\text { work }\end{array}$ \\
\hline
\end{tabular}

\section{Conclusions}


The proposed biosensor is numerically simulated and performance parameters is also analyzed for the bimetallic $(\mathrm{Au}) / \epsilon-\mathrm{SnSe} / \mathrm{graphene}$ nanosheet based modified Kretschmann configuration. The study is carried out at $633 \mathrm{~nm}$ operating wavelength, and highest sensitivity $214 \mathrm{deg} / \mathrm{RIU}$ is obtained for the mono layers of the $\epsilon-$ SnSe allotrope and graphene. The thickness of the gold layers is 40 and $20 \mathrm{~nm}$ respectively is taken in this work. The refractive index of the medium is taken as 1.368. Proposed sensor is useful for the study of the chemical, environment, biomolecule and analytes.

\section{Declarations}

Code Availability: Not applicable.

Funding Information: No funding available

Conflicts of interest/Competing interests: The authors declare that they have no conflict of interest.

Availability of data and material: No data available.

\section{Authors' contributions}

NS Formulated the problem statement wherein giving the theoretical background for SPR biosensor. He also helped in drafting the manuscript.

BK formulated the problem statement and improvised the quality of the figure and graph.

KPR provided the theoretical background to biosensing and the importance of Optical Biosensing. $\mathrm{He}$ also helped in reviewing the manuscript.

AJ provided statistical analysis for the results. He provided the theoretical background to SPR biosensors.

AP provided statistical analysis for the results. He provided the theoretical background to SPR biosensors. He also helped in reviewing and formatting the manuscript.

\section{Ethics approval}

Not applicable. The work presented in this manuscript is mathematical modelling only for the proposed biosensor. No experiment was performed on the human body and/or living organism/ animal. So, an ethical approval from an ethical committee is not required.

\section{Consent to participate}

All the author willing to participate in the work presented in this manuscript. On the behalf of all author, communicating author is authorized to give their consent.

\section{Consent for publication}


All the authors have given their consent to publish this work.

\section{References}

1. Bellassai N, D'Agata R, Jungbluth V, Spoto G, "Surface Plasmon Resonance for Biomarker Detection: Advances in Non-invasive Cancer Diagnosis," Front. Chem., vol. 7, no. August, pp. 1-16, 2019

2. Asif M et al., "The role of biosensors in coronavirus disease-2019 outbreak," Curr. Opin. Electrochem., vol. 23, no. January, pp. 174-184, 2020

3. Nguyen HH, Park J, Kang S, Kim M (2015) Surface plasmon resonance: A versatile technique for biosensor applications. Sensors 15(5):10481-10510

4. Taha BA et al (2020) An Analysis Review of Detection Coronavirus Biosensor Application. Sensors 20(23):6764

5. Mauriz E (2020) Recent progress in plasmonic biosensing schemes for virus detection. Sensors (Switzerland) 20(17):4745

6. Ahmed A, Rushworth JV, Hirst NA, Millner PA (2014) Biosensors for whole-cell bacterial detection. Clin Microbiol Rev 27(3):631-646

7. Bhalla N, Jolly P, Formisano N, Estrela P (2016) Introduction to biosensors. Essays Biochem 60(1):18

8. Hossain MB, Tasnim T, Abdulrazak LF, Rana MM, Islam MR (2020) A Numerical Approach to Design the Kretschmann Configuration Based Refractive Index Graphene-MoS2 Hybrid Layers With TiO2SiO2 Nano for Formalin Detection. Photonic Sensors 10(2):134-146

9. Akowuah EK, Gorman T, Haxha S (2009) Design and optimization of a novel surface plasmon resonance biosensor based on Otto configuration. Opt Express 17(26):23511

10. Li L et al (2017) Dual Kretschmann and Otto configuration fiber surface plasmon resonance biosensor. Opt Express 25(22):26950

11. Pal A et al., "Prism Based Surface Plasmon Resonance Biosensor for Biomedical Applications," in ICOL-2019, Springer Proceedings in Physics 258, Springer Singapore, 2020, pp. 1-4

12. Zeng $Y$ et al (2020) Phase interrogation SPR sensing based on white light polarized interference for wide dynamic detection range. Opt Express 28(3):3442

13. Singh Y, Raghuwanshi SK (2019) Electromagnetic wave sensors Sensitivity Enhancement of the Surface Plasmon Resonance Gas Sensor With Black Phosphorus. IEEE Sensors Lett 3(12):1-4

14. Pal A, Jha A, "A theoretical analysis on sensitivity improvement of an SPR refractive index sensor with graphene and barium titanate nanosheets," Optik (Stuttg)., vol. 231, no. 166378, 2021

15. Homola J (2003) Present and future of surface plasmon resonance biosensors. Anal Bioanal Chem 377(3):528-539

16. Zhao LD et al (2014) Ultralow thermal conductivity and high thermoelectric figure of merit in SnSe crystals. Nature 508(7496):373-377 
17. Hu ZY, Li KY, Lu Y, Huang Y, Shao XH (2017) High thermoelectric performances of monolayer SnSe allotropes. Nanoscale 9(41):16093-16100

18. Zhao LD et al., "Ultrahigh power factor and thermoelectric performance in hole-doped single-crystal SnSe," Science (80-).,, vol. 351, no. 6269, pp. 141-144, 2016

19. Verma A, Prakash A, Tripathi R (2015) Performance analysis of graphene based surface plasmon resonance biosensors for detection of pseudomonas-like bacteria. Opt Quantum Electron 47(5):1197-1205

20. Sharma AK (2018) Blue Phosphorene / MoS 2 Heterostructure Based SPR Sensor With Enhanced Sensitivity. IEEE Photonics Technol Lett 30(7):595-598

21. Meshginqalam B, Barvestani J (2018) Performance Enhancement of SPR Biosensor Based on Phosphorene and Transition Metal Dichalcogenides for Sensing DNA Hybridization. IEEE Sens J 18(18):7537-7543

22. Wei PC et al (2016) The intrinsic thermal conductivity of SnSe. Nature 539(7627):E1-E2

23. Dai X, Liang Y, Zhao Y, Gan S, Jia Y, Xiang Y (2019) Sensitivity enhancement of a surface plasmon resonance with tin selenide (SnSe) allotropes. Sensors (Switzerland) 19(1):1-8

24. Amendola V, Pilot R, Frasconi M, Maragò OM, latì MA (2017) Surface plasmon resonance in gold nanoparticles: A review. J Phys Condens Matter 29:no. 20

25. Rathi S et al (2015) Tunable Electrical and Optical Characteristics in Monolayer Graphene and FewLayer MoS2 Heterostructure Devices. Nano Lett 15(8):5017-5024

26. Aksimsek S, Jussila H, Sun Z (2018) Graphene - MoS 2 - metal hybrid structures for plasmonic biosensors. Opt Commun 428:233-236

27. Mudgal N, Singh PYJAG, "BaTiO 3 -Graphene-Affinity Layer - Based Surface Plasmon Resonance (SPR) Biosensor for Pseudomonas Bacterial Detection," Plasmonics, 2020

28. Zhang N, Han C, Fu X, Xu YJ (2018) Function-Oriented Engineering of Metal-Based Nanohybrids for Photoredox Catalysis: Exerting Plasmonic Effect and Beyond. Chem 4(8):1832-1861

29. Mathur D, Medintz IL, "The Growing Development of DNA Nanostructures for Potential HealthcareRelated Applications," Adv. Healthc. Mater., vol. 8, no. 9, 2019

30. Ritzefeld M, Sewald N, "Real-Time Analysis of Specific Protein-DNA Interactions with Surface Plasmon Resonance," J. Amino Acids, vol. 2012, pp. 1-19, 2012

31. Terao K et al (2012) Size-exclusion SPR sensor chip: Application to detection of aggregation and disaggregation of biological particles. Analyst 137(9):2192-2198

32. Srivastava A, Prajapati YK (2020) Effect of sulfosalt and polymers on performance parameter of SPR biosensor. Opt Quantum Electron 52(10):1-14

33. Pang K, Dong W, Zhang B, Zhan S, Wang X (2016) A Performance-Enhanced Bimetallic Chip for the Detection of Cadmium Ions with Surface Plasmon Resonance. Plasmonics 11(4):1119-1128

34. Brahmachari K, Ray M (2013) Effect of prism material on design of surface plasmon resonance 
35. Gupta BD, Verma RK, "Surface Plasmon Resonance-Based Fiber Optic Sensors: Principle, Probe Designs, and Some Applications," J. Sensors, vol. 2009, pp. 1-12, 2009

36. Murata H, Saitoh N, Yoshizawa N, Suemasu T, Toko K (2017) High-quality multilayer graphene on an insulator formed by diffusion controlled Ni-induced layer exchange. Appl Phys Lett 111(24):3-7

37. Varasteanu P (2021) Optimizing the Tin Selenide (SnSe) Allotrope/Gold-Based Surface Plasmon Resonance Sensors for Enhanced Sensitivity. Plasmonics 16(2):341-347

\section{Figures}

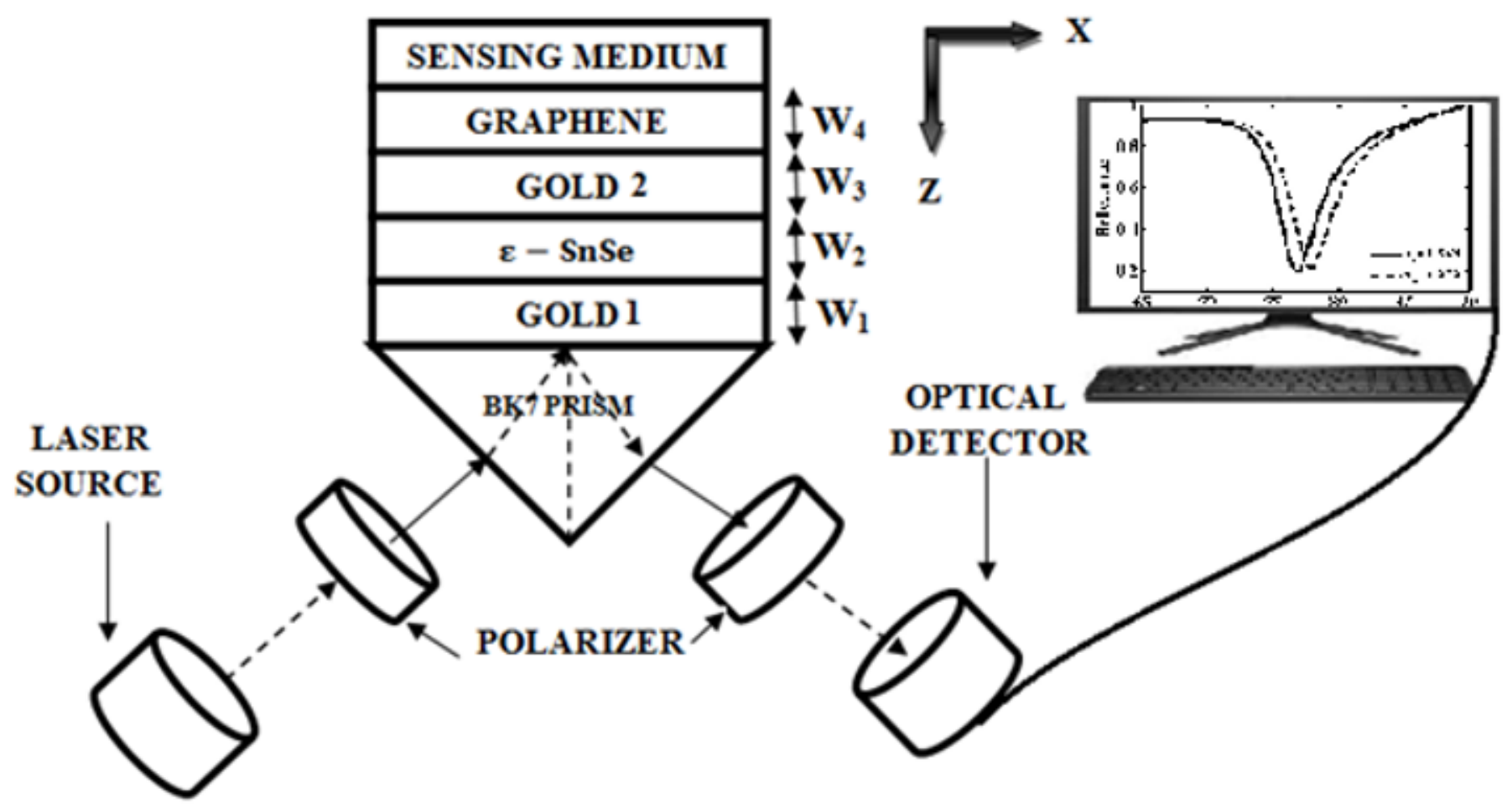

Figure 1

Heterostructure design of the proposed biosensor 


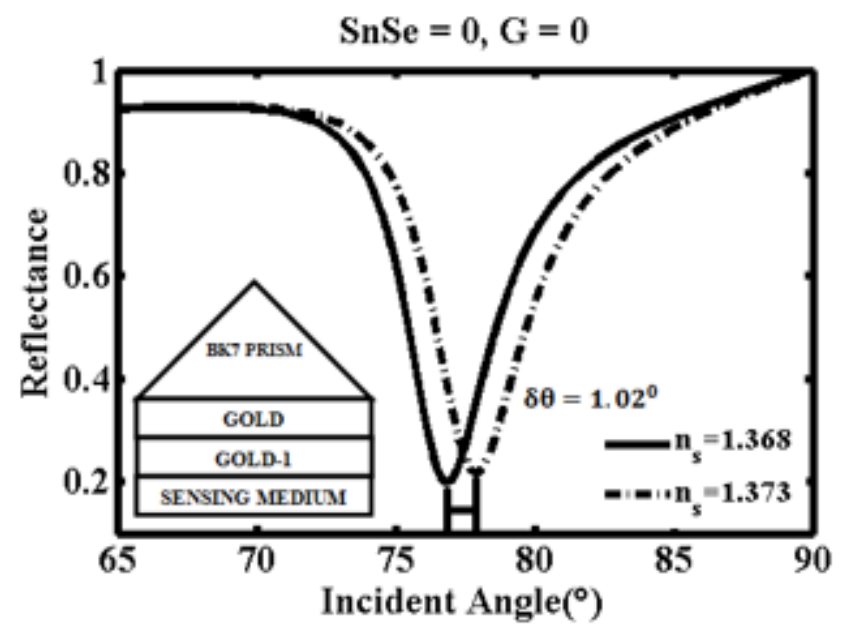

(a)

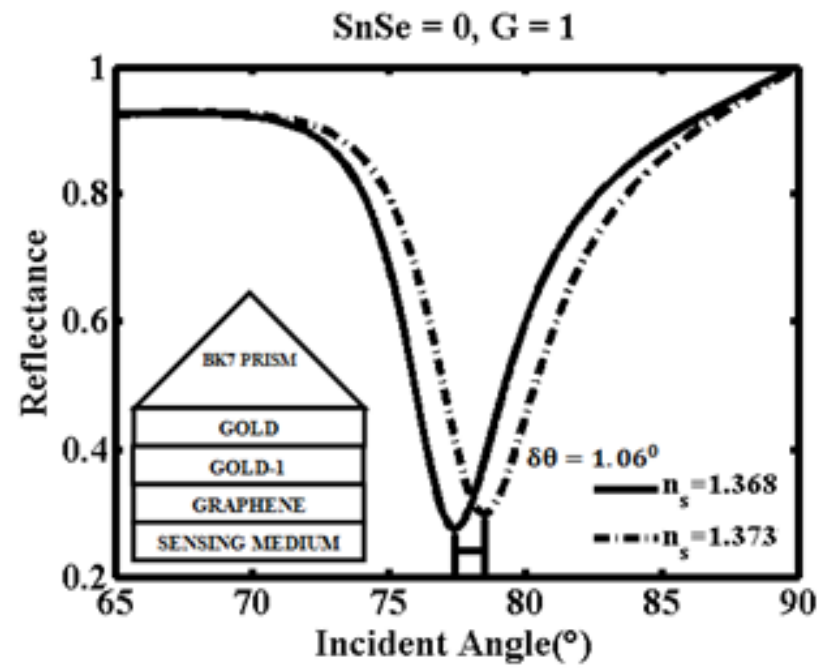

(c)

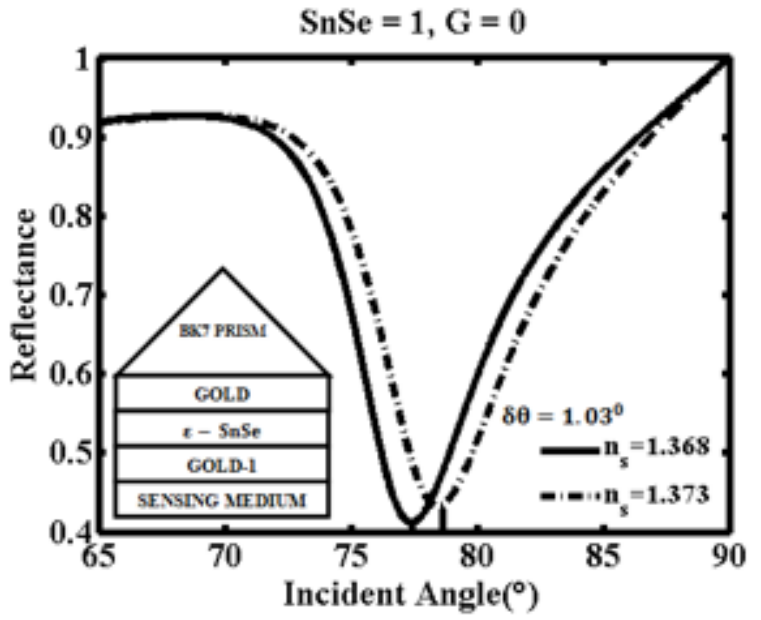

(b)

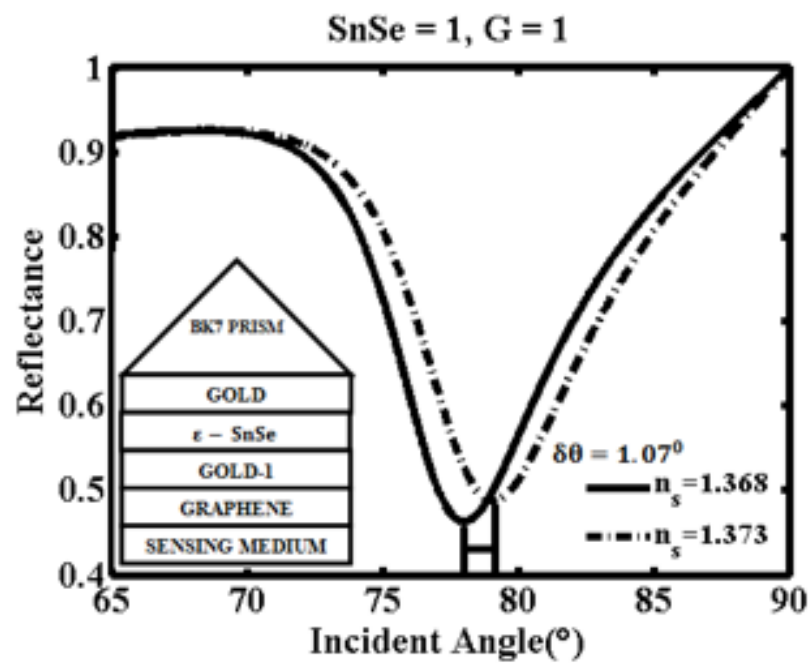

(d)

Figure 2

Reflectance vs. incident angle (o) at the same RI change with $\varepsilon$-SnSe and Graphene (a) SnSe=0,G=0, (ii) $\mathrm{SnSe}=0, G=1$, (iii) $\mathrm{SnSe}=1, \mathrm{G}=0$, (iv) $\mathrm{SnSe}=1, G=1$ 


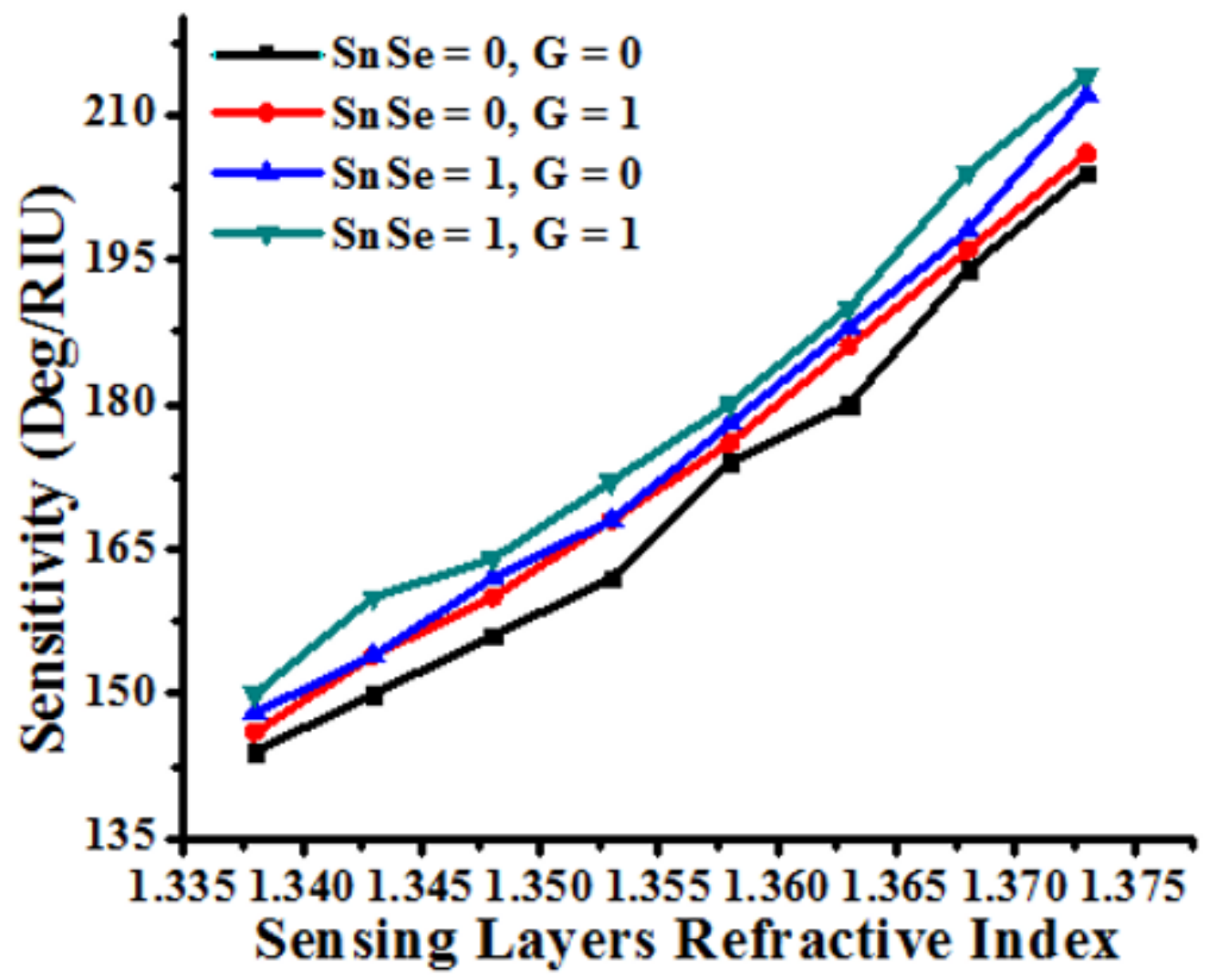

Figure 3

Plot of Sensitivity (Deg/RIU) vs. Sensing layers refractive index

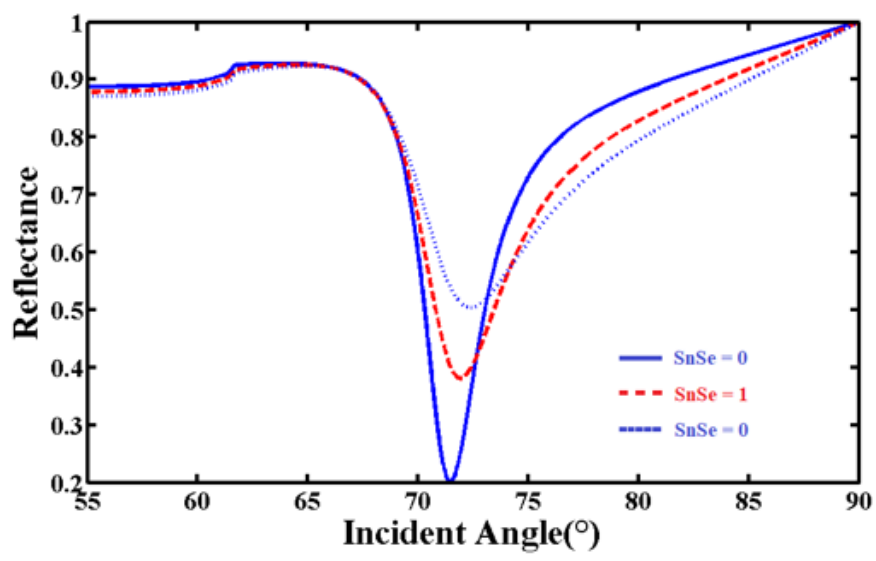

(a)

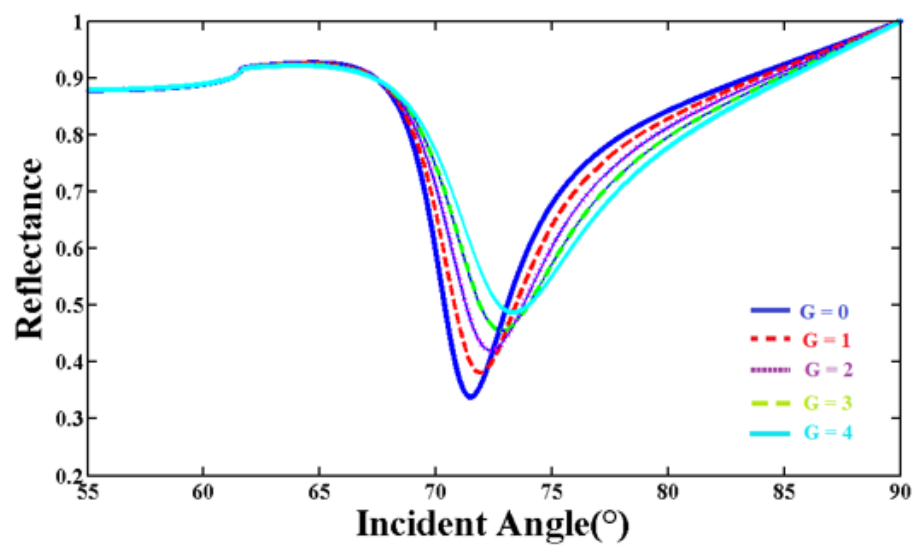

(b)

\section{Figure 4}

(a), (b) Change in the reflectance w.r.t incident angle (a) variation in the number of tin selenide layers at one graphene layer and (b) variation in the number of graphene layers at one tin selenide. 


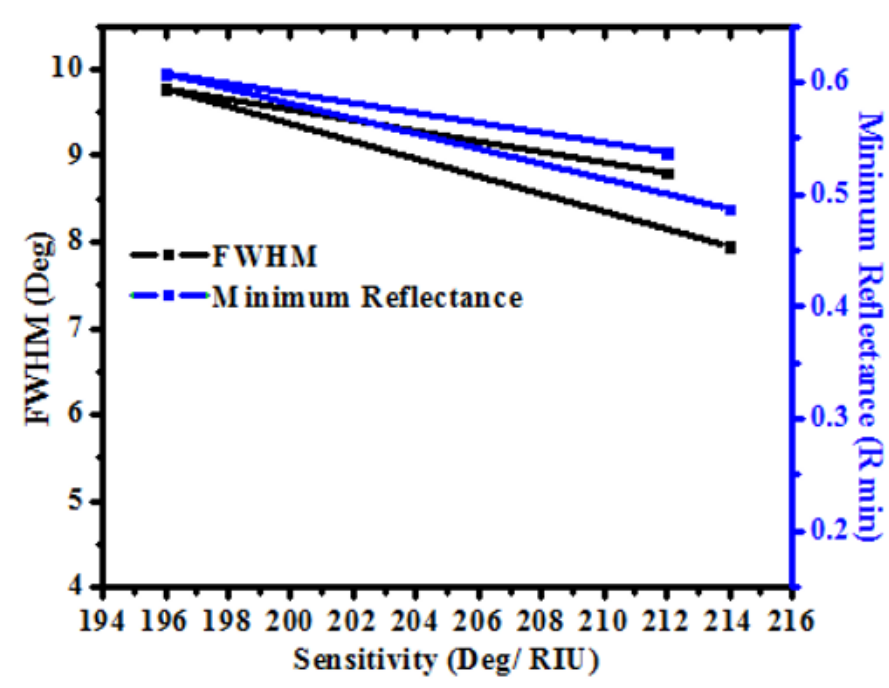

(a)

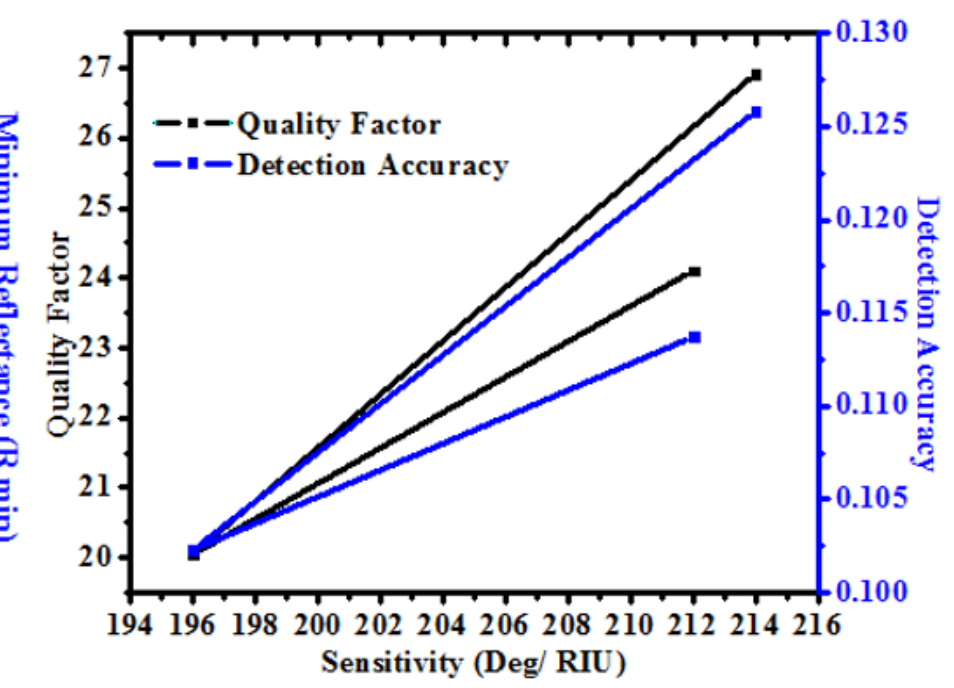

(b)

\section{Figure 5}

Graphical representation comparing (a) the sensitivity with FWHM and Minimum Reflectance of $\varepsilon$-SnSe layers and graphene layer, (b) the sensitivity with Quality Factor and Detection Accuracy of $\varepsilon$-SnSe layers and graphene layer 